\title{
Parallelization of Module Network Structure Learning and Performance Tuning on SMP
}

\author{
Hongshan Jiang ${ }^{1}$, Chunrong Lai ${ }^{2}$, Wenguang Chen ${ }^{1}$, \\ Yurong Chen ${ }^{2}$, Wei $\mathrm{Hu}^{2}$, Weimin Zheng ${ }^{1}$, and Yimin Zhang ${ }^{2}$ \\ ${ }^{1}$ Tsinghua University \\ Dept. of Computer Science \\ Beijing, 100084 China \\ jhs03@mails.tsinghua.edu.cn \\ $\{$ cwg, zwm-des $\} @$ tsinghua.edu.cn \\ ${ }^{2}$ Intel China Research Center Ltd. \\ 9/F, Raycom Infotech Park A, Zhong Guan Cun \\ Beijing, 100080 China \\ \{chunrong.lai, yurong.chen, wei.hu, \\ yimin.zhang\}@intel.com
}

\begin{abstract}
As an extension of Bayesian network, module network is an appropriate model for inferring causal network of a mass of variables from insufficient evidences. However learning such a model is still a timeconsuming process. In this paper, we propose a parallel implementation of module network learning algorithm using OpenMP. We propose a static task partitioning strategy which distributes sub-search-spaces over worker threads to get the tradeoff between loadbalance and software-cache-contention. To overcome performance penalties derived from shared-memory contention, we adopt several optimization techniques such as memory pre-allocation, memory alignment and static function usage. These optimizations have different patterns of influence on the sequential performance and the parallel speedup. Experiments validate the effectiveness of these optimizations. For a 2,200 nodes dataset, they enhance the parallel speedup up to $88 \%$, together with a $2 X$ sequential performance improvement. With resource contentions reduced, workload imbalance becomes the main hurdle to parallel scalability and the program behaviors more stable in various platforms.
\end{abstract}

\section{Introduction}

In recent years, Bayesian networks [10] have been widely applied in various fields such as bioinformatics,

\footnotetext{
${ }^{0}$ Supported by Intel Corporation, the National Natural Science Foundation of China under Grant No. 60273007 and the ChinaGrid.
}

speech processing and text mining to represent probabilistic influences among stochastic variables, for instance to identify gene regulatory networks from microarray data. In real world applications, it is often the case that the problem domain is so complex that it contains a mass of variables while there are only a few instances available due to expensive cost of acquiring data. In these cases, the amount of data is insufficient to learn the underlying distribution: statistical noises tend to lead spurious dependencies that significantly overfit the data. To fit with these cases, an extension of traditional Bayesian network, namely module network [11], was introduced.

Module network can be viewed as a Bayesian network in which variables in the same module share the same parents and conditional probability distribution. A real world example corresponding to this model is that many genes in a cell are co-regulated by the same factors and exhibit the same expression pattern. Module network introduces the clustering concept into regular Bayesian network and make up its aforementioned defect. Currently it has been successfully used in Gene regulatory analysis [12].

Compared with traditional Bayesian network structure learning $[10,8]$, module network structure learning has a further structure to learn, namely module assignment. Once the assignment is determined, learning dependency structure of a module network is like that of a Bayesian network except that the targets are changed from nodes to modules. Module network significantly reduces the model complexity and the number of parameters, however learning such a model is still a computation-intensive and time-consuming process. 
A parallel learning program of module network on distributed memory multiprocessor systems was recently implemented and optimized [9], but as far as we know the parallel version for shared-memory multiprocessor systems has not appeared. We see that the behaviors of parallel programs on shared-memory multi-processors are more difficult to understand. It is mainly due to the facts that there are more types of resource contentions in SMP and communications between processors are implicit. Because of the abundance of SMP systems in recent years and the trend of on-chip multi-core systems in the future [7], implementing such a hotspot application on SMP systems and analyze its characteristics is of great importance.

In this paper, as our main contribution, we propose a parallel module network learning algorithm with OpenMP [4] and present a set of recipes to cope with performance penalties that arise from resource contentions. Experiments show that these optimizations have sound effectiveness.

The rest of this paper is organized as follows. In section 2, we describe a hill climbing algorithm for learning module network. In section 3 , the parallelized algorithm is proposed. In section 4, measurements of hotspots and thread profiles by Intel $\mathbb{R}$ VTune $^{\text {TM }}[1]$ together with corresponding analysis are presented. In section 5, we give some optimization techniques to deal with memory sharing. Experiment results are given and discussed in section 6 . In the last section, we give the summary and future works.

\section{Module Network Learning Algorithm}

Module network structure learning is an optimization problem, in which a very large search space must be explored to find the optimal solution. Because a brutal search will lead to super-exponential computational complexity, we use a greedy hill climbing algorithm to find a local optimal solution.

The hill climbing algorithm first clusters all nodes into modules, none of which has parent nodes. This initial module network model is treated as the start point in the search space (solution space). The algorithm searches all the nearest neighbors of the start point in the search space, then selects the neighbor that has the highest score as the new start point. This procedure iterates until no neighbor has higher score than the current start point (i.e., it has reached a local optimal). Here the "neighbors" of a module network model are of two kinds: dependency neighbors and assignment neighbors. The former can be generated from the current model by adding or deleting a single arc, subject to the acyclic constraint; the latter can be gen- erated from the current model by moving a node from a module to another, subject to the acyclic constraint and without changing module count.

Learning dependency structure and learning module assignment are equally important, so every iteration step combines searches for dependency neighbors and searches for assignment neighbors. In our implementation, these searches are organized as two iterative phases. The algorithm is presented in Figure 1.

Bayesian Information Criteria (BIC) [8] score metric is used in the algorithm for scoring a module network model. It equals to the log likelihood of the data subtract the penalty of the model's complexity. A model $\mathrm{M}$ has BIC score with dataset $\mathrm{D}$ as below:

$$
\operatorname{Score}(M, D)=\log (P(D \mid M))-0.5 \times \log (E) \times C(M)
$$

where $P(D \mid M)$ is the probability that data $D$ is generated from model $\mathrm{M}$; $\mathrm{E}$ is the number of evidences in the dataset $\mathrm{D}$, and $\mathrm{C}(\mathrm{M})$ is a constant that describes the complexity of model M. From the formula, we can see that the main computation to get a score is to compute the probability $\mathrm{P}(\mathrm{D} \mid \mathrm{M})$. According to the conditional

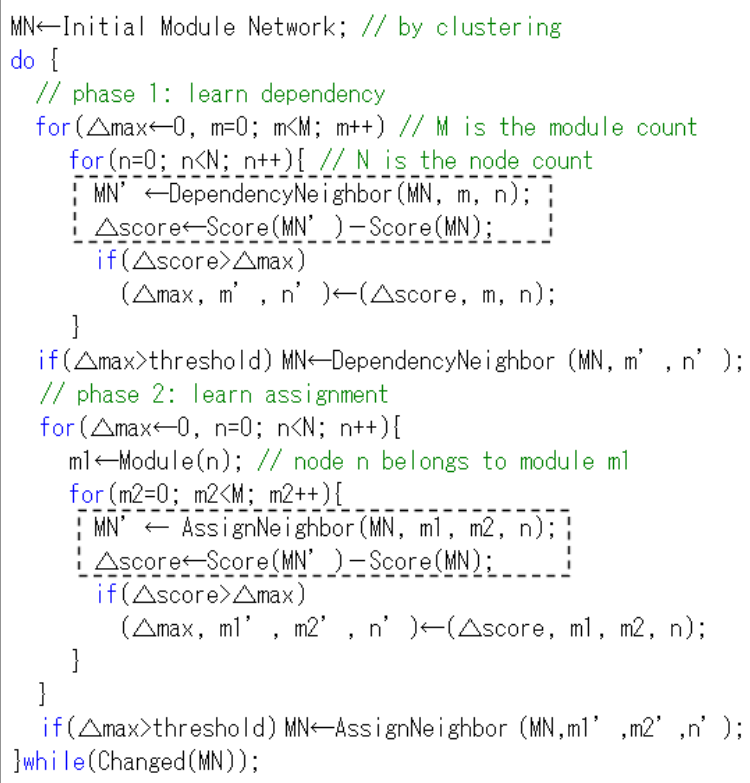

\section{Figure 1. Sequential algorithm}

independence in Bayesian network, the total score of a model can be decomposed into the sum of its families' scores:

$$
\operatorname{Score}(M, D)=\sum_{i} \operatorname{Score}\left(\text { Family }_{i}, D\right)
$$

Different from that in traditional Bayesian network model, here a family consists of a module (a set of child 
nodes) and its parent nodes. Since each family's score will not change once it has been computed out, we use a cache to store each family's score after the score has been computed. Consequently when we need to compute a family's score, we look for it in the cache first. If cache hit, we load score from cache directly; otherwise, when encounter a new family, we compute the score from scratch and save it into cache for possible future use.

From Equation 2, we infer that the delta score between a neighbor and the current model equals to the delta score of the changed family. Therefore the problem of computing score of each neighbor model is changed to computing scores of neighbor families.

Analyze the computational complexity of this algorithm is pretty hard due to the search path is tightly correlated to the training dataset and the initial clustering. In addition, using of score caches complicated the situation. In [9] an approximate estimation is given.

An implementation of the sequential module network learning algorithm is available in the Probabilistic Network Library (PNL) [2]. It constructs the start point of our work.

\section{Workload Parallelization}

To parallelize the hill-climbing structure learning algorithm, we first identify the most computational intensive modules, finding parallel opportunities within it. By using VTune ${ }^{\mathrm{TM}}$ performance analyzer, we find most of the execution time is spent on computing scores of neighbor families. On the other hand, each family score can be computed independently. Therefore each family score computation becomes a single workload unit for parallelization.

Neglecting score cache factor, the workload distribution of family score computations is inherently not even. Computing scores of neighbor families from the same family have close workloads while computing scores of neighbor families from different families may vary greatly in workloads.

Unfortunately the introduction of score caches exaggerates this workload imbalance. In all rounds except the first round, only newly encountered neighbor families need to be computed their scores from scratch, other neighbor families' scores can be obtained from score caches. The newly encountered neighbor families include those neighbors of the just changed family of current model in the previous round and a few neighbors of the unchanged family due to the possible release of some acyclic constraint.

In our implementation, score caches are saved as sparse matrix elements and are indexed by family parents' numbers. Family children's (nodes inside the module) numbers are not used as indices for two reasons. The first is that higher index dimensions lead to more performance penalty when retrieving content from sparse matrix. The second is that computation of delta score of changing children of a family has much lower complexity than that of changing parents of a family. As a result, every sparse matrix is bound to a module. In addition, the score caches are only used in the dependency-learning phase which makes choice by altering parents. In assignment-learning phase, delta score of neighbor families are computed directly.

Now we talk about issues related to parallelization.

Granularity. If we choose to partition each family score computation into tasks, the granularity is fine but the parallelism and synchronization overhead become heavy. Otherwise choosing to parallelize with module granularity will lead to intolerable workload imbalance. As a trade-off, we treat each family score computation as a single workload unit in dependency-learning phase, while treat delta score computations of those neighbor families derived from moving one node as a single workload unit in assignment-learning phase.

Scheduling. In dependency-learning phase we notice that resource contention caused by sharedmemory usage hinder us from using a workload balanced scheduling strategy. Sparse matrices for caching scores are implemented as hash tables with linked lists, which makes each sparse matrix a critical resource. But from the second round of learning process, fromscratch computations concentrate in one module (i.e. related to the same sparse matrix). So synchronization overhead for using critical section will be heavy. Therefore we make a copy of sparse matrices for every worker thread. At this point, we choose static scheduling strategy so that the search space mapped in each spare matrix can be divided into fixed nonoverlapped sub-spaces which are thereafter assigned to different worker threads. To use the common acyclic judgment, actually we group nodes according to the module they belong to and then schedule workloads statically. This makes things more complex. But as in one round of the iteration process at most two modules change their components by one node, the nonoverlapping feature of sub-spaces is maintained in an approximate way. In assignment-learning phase we simply use a dynamic scheduling strategy to gain good workload balance.

Synchronization. As OpenMP only support simple reduction variable, we set $\Delta$ max and related indices as threadprivate variables. Each worker thread compute its local maximum score in $\Delta \max$, then in the critical section at the end of each phase, the global 
maximum score is collected.

Figure 2 illustrates the parallelized algorithm.

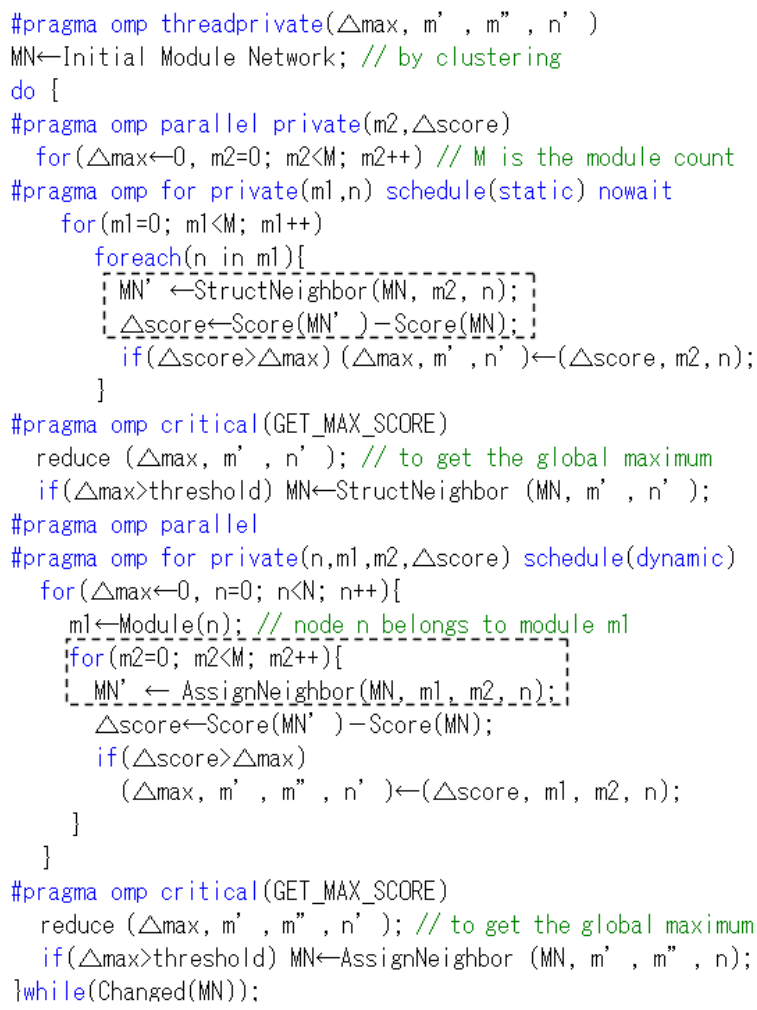

Figure 2. Parallel algorithm using OpenMP

\section{First Round Performance Analysis}

\subsection{Baseline Program and Hardware}

The first group of issues before the performance tuning process are the baseline construction and hardware settings.

According to the descriptions in section 3 we developed a package, which includes high level optimizations like common sub-expression elevation and redundancy elimination [5]. We compile this package with options "-O3 -openmp", hence constructed our baseline program.

The hardware platforms are two Xeon ${ }^{\mathrm{TM}}$ SMP machines. One is a 4-way multiprocessor system named QP (quad-processors) machine, the other is a UnisysEs7000 system which consists of 16 processors. Their key characteristics are listed in Table 1.

Processor prefetcher settings are important hardware settings which can influence application performance. Whether or not to set a prefetcher on should

\begin{tabular}{|c|c|c|}
\hline Platform & QP-machine & Unisys-Es7000 \\
\hline Processor & 4 -way $2.8 \mathrm{GHz}$ & 16 -way $3.0 \mathrm{GHz}$ \\
\hline $\begin{array}{l}\text { L1 d- } \\
\text { cache }\end{array}$ & $\begin{array}{l}8 \mathrm{~KB}, \text { hit latency } \\
2 \text { cycles }\end{array}$ & $\begin{array}{l}8 \mathrm{~KB} \text {, hit latency } \\
2 \text { cycles }\end{array}$ \\
\hline $\begin{array}{l}\text { L2 } \\
\text { cache }\end{array}$ & $\begin{array}{l}512 \mathrm{~KB}, \quad \text { hit la- } \\
\text { tency } \sim 10 \text { cycles }\end{array}$ & $\begin{array}{l}512 \mathrm{~KB}, \quad \text { hit la- } \\
\text { tency } \sim 10 \text { cycles }\end{array}$ \\
\hline $\begin{array}{l}\text { L3 } \\
\text { cache }\end{array}$ & $\begin{array}{l}2 \mathrm{MB} \text {, hit latency } \\
30+\text { cycles }\end{array}$ & $\begin{array}{l}4 \mathrm{MB} \text {, hit latency } \\
30+\text { cycles }\end{array}$ \\
\hline $\begin{array}{l}\text { L4 } \\
\text { cache }\end{array}$ & None & $\begin{array}{l}32 \mathrm{MB} \text { on-board, } \\
300+\text { cycles }\end{array}$ \\
\hline FSB & $400 \mathrm{MHz}$ & $400 \mathrm{MHz}$ \\
\hline Interconn. & FSB & Crossbar \\
\hline Memory & $\begin{array}{l}2 \mathrm{~GB} \text {, peak band- } \\
\text { width } 2.1 \mathrm{~GB} / \mathrm{s}\end{array}$ & $\begin{array}{l}\text { 8GB, peak band- } \\
\text { width } 3.2 \mathrm{~GB} / \mathrm{s}\end{array}$ \\
\hline$\overline{\mathrm{OS}}$ & Linux 2.4.20smp & Linux 2.4.20smp \\
\hline
\end{tabular}

Table 1. Hardware configuration

be determined by memory access pattern of the target application. From analysis in section 3, we know that accessing score caches will lead to irregular memory access pattern, since score caches are implemented as items in hash table. On the other hand, when computing family score from scratch, the matrix access stride has regular pattern. Therefore we need some quantitative measurements to judge which has dominant impact.

In Intel's Pentium ${ }^{\mathrm{TM}} 4$ and Xeon ${ }^{\mathrm{TM}}$ processors, there are two hardware-based prefetchers which can prefetch data from memory to cache. The first one is called stride prefetcher which tries to stay some cache lines ahead of current data access locations, the other one is called adjacent line prefetcher which fetches the adjacent cache line (in same sector). These Prefetchers settings can be changed through BIOS options.

As anticipated, measurements show that prefetcher settings do impact system performance. Table 2 lists the running time metrics of the baseline program with different prefetcher settings on these two machines, which are normalized to the running time with 4threads in the default setting of corresponding machines. The four-thread (4T) relative speedups are also listed in the table. It is seen that the benefit of the prefetchers decreases as processor numbers increase. And the best relative speedups are achieved if turning off the prefetchers. So we turn off both prefetchers in these machines for the following experiments.

After we determine the baseline program and hardware settings, it is seen that the parallel speedup varies greatly on different platforms and is not good enough on both of them. As the last line of Table 2 shows, the $4 \mathrm{~T}$ speedup is $2.56 \mathrm{X}$ on the QP-machine and $1.74 \mathrm{X}$ on Unisys-es7000. It needs to be pointed out that the aw- 


\begin{tabular}{|l|l|l|l|l|l|l|}
\hline & \multicolumn{3}{|c|}{ QP-machine } & \multicolumn{3}{c|}{ Unisys-Es7000 } \\
\hline St./Ad. & seq. & Par. & Sp. & Seq. & Par. & Sp. \\
\hline on/on & 2.37 & 1.02 & 2.33 & 1.70 & 1.05 & 1.62 \\
\hline on/off & 2.38 & 0.95 & 2.50 & 1.71 & 0.99 & 1.73 \\
\hline off/on & 2.42 & $\mathbf{1 . 0 0}$ & 2.42 & 1.72 & 1.11 & 1.55 \\
\hline off/off & 2.47 & 0.96 & $\mathbf{2 . 5 6}$ & 1.74 & $\mathbf{1 . 0 0}$ & $\mathbf{1 . 7 4}$ \\
\hline
\end{tabular}

Table 2. Normalized running time and $4 \mathrm{~T}$ speedups with prefetcher settings

ful performance is not result from the parallelization strategies. In fact, both theoretical analysis (in section 3 ) and profiling (in section 4.2) confirm that we have a relatively optimum version according to high-level task partition.

\subsection{Thread Profile and Hotspot Functions}

To gain the running metrics of our program and measure which parts influence the whole performance to what extent, we use VTune ${ }^{\mathrm{TM}}$ analyzer, which consists of thread profiling and sampling profiling, to get thread profile and hotspot functions.

The thread profiling process creates thread profile which can be expected to give hints to understand or optimize the parallel speedup. The process is based on the instrumentation. The codes are in fact recompiled with -openmp_profile instead of -openmp. Instrument codes are added to the OpenMP pragma or even the Pthreads/Winthreads primitives. Finally by running those instrumented binary, VTune can give a report of run-time ratio of the parallel-part, sequential-part, load-imbalance, barrier as well as the synchronization percentage and the overheads ratio. In our experiments the thread profile only increases $5 \% \sim 8 \%$ overhead to the program. So that the gained thread profile of baseline program is trustworthy.

\begin{tabular}{|l|l|l|l|l|l|}
\hline & $\begin{array}{l}\text { Para. } \\
\text { ratio }\end{array}$ & $\begin{array}{l}\text { Seq. } \\
\text { ratio }\end{array}$ & $\begin{array}{l}\text { Imba. } \\
\text { / barr. }\end{array}$ & $\begin{array}{l}\text { Locks } \\
\text { / Sync. }\end{array}$ & $\begin{array}{l}\text { Over- } \\
\text { heads }\end{array}$ \\
\hline QP & $94.0 \%$ & $0.5 \%$ & $4.1 \%$ & $0.9 \%$ & $0.4 \%$ \\
\hline Uni. & $90.1 \%$ & $0.3 \%$ & $6.5 \%$ & $0.5 \%$ & $0.3 \%$ \\
\hline
\end{tabular}

Table 3. Thread profile of the baseline

Table 3 lists the thread profiles. We found that when running in parallel most of the time spends on the parallel part. The most serious parallelism-limiting factor is the imbalance part that arises from the LearnDependency function because of the static scheduling strategy there. But the number $4.1 \%$ or $6.5 \%$ does not occupy a large ratio. At this way the parallel speedup should be much better than what we got. As we have seen that the aggregate running time increases much from uni-thread (UT) running to multi-threads (MT) running. It is highly possible that some operations run much slower in multi-processors configuration than in uni-processor configuration.

In the next step, we switch to VTune sampling process to get bottlenecks on run time. In this process, interrupts are generated on every interval of given number of hardware events based on hardware counters, and then samples are noted down on current PC. Thus the sampling process can give a run-time hotspot instructions distribution for interested events. When the binary is built with debug information, for example built with -g in gcc or -zi in Visual studio compiler or in icc compiler, the distribution can be seen in higher level such as function-level, class-level or module-level.

In Table 4, we list those hotspot functions which cost up to $90 \%$ of the running time on both machines with one thread and four threads, respectively. These hotspot functions can be divided into four modules, as Table 4 shows. The first module is ModuleNet, which contains the main part of functions for learning module network. Among them, function ScoreCacheAccess accesses score caches; function ProcessingStatisticalData, which include log computations, computes score from the sufficient statistics [8]. The second module is Libpthread.so, which deals with primitive operations of pthead. The third module is Libc-2.3.2.so, which is mainly about memory operations. The last module is libguide.so, which include those part of OpenMP library calls.

Neglecting libguide.so, which relates to OpenMP runtime environment, we can see from Table 4 that vector functions such as push_back, erase, assign etc. significantly increase their percentage as thread count increased. Other functions such as ScoreCacheAccess and free have the same tendency but less degree. The scalability problem in vector functions, malloc and free give us hints to think about the cost of dynamic memory allocation/release. While the prolonged accessing time of ScoreCacheAccess makes us to pay attention to memory layout [5].

\subsection{Memory Hierarchy Statistics}

For further assurance, we get the memory-hierarchy micro-architectural statistics with VTune ${ }^{\mathrm{TM}}$ in Table 5. As this table shows, the Level 1 cache misses, FSB (front-side-bus) activities, 64KB aliasing, and DTLB (Data Translation Look-aside Buffer) page walks all increase from $1 \mathrm{~T}$ to $4 \mathrm{~T}$. These data confirm that the memory behavior in MP (Multi-Processors) case is not 


\begin{tabular}{|c|c|c|c|c|}
\hline $\begin{array}{l}\text { Module name / } \\
\text { Function name }\end{array}$ & $\begin{array}{l}\text { QP- } \\
1 \mathrm{~T}\end{array}$ & $\begin{array}{l}\text { QP- } \\
4 \mathrm{~T}\end{array}$ & $\begin{array}{l}\text { Uni.- } \\
1 \mathrm{~T}\end{array}$ & $\begin{array}{l}\text { Uni.- } \\
4 \mathrm{~T}\end{array}$ \\
\hline ModuleNet & $48.6 \%$ & $\uparrow 61.9 \%$ & $46.4 \%$ & $43.2 \%$ \\
\hline \begin{tabular}{l|l} 
& ScoreCacheAccess
\end{tabular} & $2.7 \%$ & $\uparrow 3.6 \%$ & $3.9 \%$ & $\uparrow 5.0 \%$ \\
\hline $\begin{array}{l}\text { ProcessingStatis- } \\
\text { ticalData }\end{array}$ & $8.4 \%$ & $2.8 \%$ & $7.8 \%$ & $3.2 \%$ \\
\hline Vector functions & $2.0 \%$ & $\uparrow 15.8 \%$ & $1.7 \%$ & $\uparrow 12.9 \%$ \\
\hline Libpthread.so & $32.2 \%$ & $22.7 \%$ & $31.2 \%$ & $22.7 \%$ \\
\hline Libc-2.3.2.so & $10.0 \%$ & $8.6 \%$ & $11.4 \%$ & $\uparrow 23.5 \%$ \\
\hline _malloc & $3.8 \%$ & $2.7 \%$ & $4.3 \%$ & $\uparrow 13.1 \%$ \\
\hline _free & $2.3 \%$ & $\uparrow 3.3 \%$ & $3.7 \%$ & $\uparrow 8.6 \%$ \\
\hline Libguide.so & & $6.2 \%$ & & $9.6 \%$ \\
\hline
\end{tabular}

Table 4. The hotspot functions

as good as in UP (Uni-Processor) case. It gives the clue that memory contention of multi-processors may be the main bottleneck of workload scalability.

\begin{tabular}{|l|l|l||l|l|}
\hline & $\begin{array}{l}\text { QP- } \\
1 \mathrm{~T}\end{array}$ & $\begin{array}{l}\text { QP- } \\
4 \mathrm{~T}\end{array}$ & $\begin{array}{l}\text { Uni.- } \\
1 \mathrm{~T}\end{array}$ & $\begin{array}{l}\text { Uni.- } \\
4 \mathrm{~T}\end{array}$ \\
\hline Instru. Loads & $36.5 \%$ & $36.5 \%$ & $36.5 \%$ & $36.5 \%$ \\
\hline L1 miss & $2.4 \%$ & $\uparrow 3.3 \%$ & $3.3 \%$ & $\uparrow 3.6 \%$ \\
\hline L2 miss & $7.0 \%$ & $2.6 \%$ & $5.1 \%$ & $4.7 \%$ \\
\hline FSB activities & $0.6 \%$ & $\uparrow 8.8 \%$ & $0.5 \%$ & $\uparrow 9.8 \%$ \\
\hline DTLB walks & $0.11 \%$ & $\uparrow 0.13 \%$ & $0.09 \%$ & $\uparrow 0.13 \%$ \\
\hline 64KB aliasing & $2.8 \%$ & $\uparrow 4.7 \%$ & $4.3 \%$ & $\uparrow 4.6 \%$ \\
\hline
\end{tabular}

Table 5. Memory performance metrics

\section{Memory Issues and Optimizations}

The implementation of aforementioned parallel module network learning algorithm is based on PNL [2], which is implemented with $\mathrm{C}++$ and STL (Standard Template Library) [3]. The push_back/erase methods of vectors in STL provide friendly interface to programmers but also implicitly use heap memory allocation/release. Due to increased competition for shared resources like the memory bandwidth and more interferes among multi-processors for cache coherence, excessive use of dynamic memory allocation/release leads to performance degrade on SMP. We used the HOARD [6] multiprocessor memory allocator trying to make those lock-free, but the performance is not improved. In the following, we will illuminate those memory issues that mostly influence the workload scalability of our program. However, it is out of the scope of this paper to describe how to optimize memory usage on SMP systems in general.

The first issue is the frequency of memory allocation and release. As memory is a shared resource in
SMP system, allocating memory for multiple concurrent threads is inefficient. Also, frequent invocations to memory allocation and release cause memory fragmentation and random memory layout. Further more, the operations are more expensive when vectors need to expand allocated space. All of these limit workload scalability.

The optimizations adopted for this issue are memory pre-allocation and static function usage. Memory pre-allocation is applied for those frequently accessed buffers or vectors. At the beginning of a code region (usually a loop region), we allocate a memory block whose length reaches the maximum length needed to run in this region. At the end of the region, we release the memory block. This remarkably reduces the frequency of memory allocation and release.

Static functions are used as substitutes for virtual functions of an object. It can reduce the overheads of construction and destruction of an object. In addition, compared with invoking virtual functions, calling static function also saves the cost of virtual tables' access. As additional costs, few more parameters need to be transferred when invoking the static functions.

The second issue is memory layout. Memory layout problem is often caused by dynamic memory allocation/release. Compiler can perform memory alignment and padding automatically for global or local variables according to compile pragma in source code. But it can not automatically align memory for dynamically allocated memory yet. This may introduce several performance issues. Intel manuals mention that the split loads or blocked store forwarding penalties due to un-alignment access take the major part of sequential penalty. Here we point out two more kinds of penalties. One is false sharing[5], which means that if the buffers are not aligned by cache line granularity, i.e. many memory lines are each partitioned by more than one processor, there will be more cache invalidates for these falsely shared data. The other kind of penalties comes from the aliasing problem. Intel reports that the processors in the test platforms have an aliasing penalty when "64 KBytes aliasing" ${ }^{1}$ exists. The reason is that the processor is doing way prediction based on that part of virtual address, so that different data with the same value for bit 0 through 15 of the linear address will compete for the same cache lines.

The optimization adopted for memory layout problem is manual memory alignment. After dynamic allocation of a large block, we compute a memory address closely after the gained memory handle, which is aligned according to the cache line size of the target

\footnotetext{
${ }^{1}$ The newer Intel processor has less aliasing penalties because there are only "4MB aliasing"
} 
architecture.

To estimate the impact of each optimization on final performance, we separate out the static-functionusage optimization and construct two optimized versions. The first one called MEMORY only uses memory pre-allocation and alignment. The second one called FINAL uses all of the aforementioned optimizations.

Although these optimizations are somewhat straightforward, they improve both the sequential performance and parallel speedup significantly. Also as we will describe in Section 6, these optimizations have different patterns of influence on the sequential performance and the parallel speedup.

\section{Experiment Results}

Experiments were carried out on the machines as described in Table 1. Hardware settings and compiler options are set as section 4.1 mentioned. The wall time was measured by computing the elapsed time between two invocations of gettimeofday, meanwhile the time for clustering the initial model were excluded from the execution time. Five measurements were made for each test case, and the average value is reported.

The dataset scaling graphs for the baseline version and the final version are shown in Figure 3. There are four candidate datasets generated from Bayesian network models which contain 1,100, 2,200, 4,400 or 8,800 nodes. It is seen that the speedups become much better in the final version. In addition, different from baseline version, whose speedup often drops with increasing dataset, the final version increases its speedup with increasing dataset. Another benefit we noticed from the two figures is that after the code optimizations the speedup variance between the two platforms becomes more similar. Thus the speedups can be understood more easily.

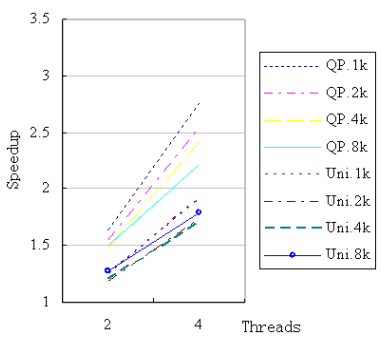

(a) baseline

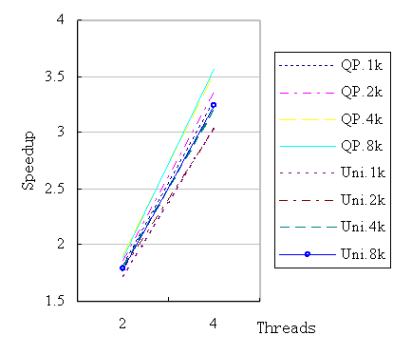

(b) after optimization

\section{Figure 3. Dataset scaling graphs}

Table 6 lists the performance metrics of our three parallel versions in the two test machines, with the dataset of 2,200 nodes grouping to 74 modules. We list the total sequential running time, the running time in the dependency-learning phases and assignmentlearning phases in Table 6(a), respectively. We also list the speedups of 4 threads in Table 6(b).

\begin{tabular}{|l|l|l|l|l|l|l|}
\hline \multicolumn{1}{|c|}{ (a) } & \multicolumn{3}{|c|}{ QP-machine } & \multicolumn{3}{c|}{ Unisys-es7000 } \\
\hline Time & Total & Dep. & Ass. & Total & Dep. & Ass. \\
\hline BASE. & 14.8 & 4.50 & 10.3 & 14.6 & 3.99 & 10.6 \\
\hline MEM. & 10.5 & 4.04 & 6.43 & 10.1 & 3.92 & 6.21 \\
\hline FINAL & 7.35 & 3.66 & 3.69 & 7.00 & 3.58 & 3.42 \\
\hline \hline \multicolumn{1}{|c|}{ (b) } & \multicolumn{3}{|c|}{ QP-machine } & \multicolumn{3}{c|}{ Unisys-es7000 } \\
\hline Sp.(4T) & Total & Dep. & Ass. & Total & Dep. & Ass. \\
\hline BASE. & 2.32 & 1.38 & 3.28 & 1.56 & 0.90 & 2.04 \\
\hline MEM. & 3.33 & 3.01 & 3.57 & 2.37 & 2.12 & 2.57 \\
\hline FINAL & 3.35 & 2.97 & 3.83 & 2.94 & 2.37 & 3.92 \\
\hline
\end{tabular}

Table 6. Performance metrics with dataset of 2,200 nodes

We deduced Table 7 from Table 6 . It lists the relative improvements of sequential performance and parallel speedup using two different optimizations, i.e. memory related optimization and static function usage.

\begin{tabular}{|l|l|l|l|l|l|l|}
\hline \multicolumn{1}{|c|}{ (a) } & \multicolumn{3}{|c|}{ QP-machine } & \multicolumn{3}{c|}{ Unisys-es7000 } \\
\hline Time\% & Total & Dep. & Ass. & Total & Dep. & Ass. \\
\hline Mem. & 41.8 & 11.4 & 61.0 & 44.3 & 1.9 & 71.2 \\
\hline Static & 42.4 & 10.3 & 74.3 & 44.7 & 9.6 & 81.4 \\
\hline \hline \multicolumn{2}{|c|}{ (b) } & \multicolumn{3}{|c|}{ QP-machine } & \multicolumn{3}{c|}{ Unisys-es7000 } \\
\hline Sp.(4T)\% Total & Dep. & Ass. & Total & Dep. & Ass. \\
\hline Mem. & 44 & 118 & 8.7 & 52 & 135 & 26 \\
\hline Static & 0.60 & -1.2 & 7.4 & 24 & 12 & 52 \\
\hline
\end{tabular}

Table 7. Relative Improvements by different optimizations

In Table 7, it is seen that the memory related optimizations improve not only the sequential performance by larger than $40 \%$, but also the relative parallel speedup by more than $40 \%$. On the other hand, static function usage results in lower parallel speedup improvement compared to its sequential counterpart. Memory related optimizations and static function usage have different effects in the two phases of the learning process, i.e. dependency-learning phase and assignment-learning phase. Memory related optimizations have lower impact on the dependency-learning phase according to the sequential performance while it has higher impact according to parallel speedup. Static function usage has consistent impact patterns with higher impact on assignment-learning phase. The reason will be described later in detail. 
From the result, we can conclude that memory related optimizations reduce not only memory accessing overheads but also interferences between processors while static function usage only reduces memory accessing overheads.

In the dependency-learning phase, using score cache makes the situation complicated. Firstly, the last line of Table 6(b) shows that although we have satisfactory parallel speedup of the assignment-learning phase in the final version, the parallel speedup of the dependency-learning phase is far from acceptable. The reason is that to avoid redundant cache computations we adopt static scheduling strategy, which results in workload imbalance. After resource contentions being reduced by aforementioned optimizations, workload imbalance becomes a major hurdle to the parallel scalability. Secondly, less sequential performance improvements are made in the dependency-learning phase, as illustrated in Table 7(a). The reason is that loading score caches, which dominates the dependency-learning phase, is rarely influenced by these optimizations in sequential mode. Thirdly, with memory optimizations we achieved remarkable improvements in the parallel speedup in dependency-learning phase, as illustrated in Table 7(b). This is because that memory alignment greatly impacts parallel behavior of loading score caches. Fourthly, static function usage only influences those from-scratch computations, which include those miss-cache computations in the dependency-learning phase and all computations in the assignment-learning phase. Therefore, more parallel speedup improvements are achieved in the assignment-learning phase by using static functions.

Further evaluation of the parallel scalability of our program on Unisys-es7000 machine was performed, with dataset of 8,800 nodes running on 16 threads. The result is consistent with the conclusion we made.

One thing we should emphasize here is that those "naïve optimizations" such as the manual redundant computation removal, computation reuse and loop unrolling [5], are observed to slightly reduce the parallel speedup. It is because that those optimizations mainly focus on hotspots, which are most likely in parallel regions of the program. Thus those optimizations lead to less parallel ratio, and finally less relative speedup according to the Amdahl's Law.

\section{Summary}

In this paper, we presented a parallel implementation of module network learning algorithm with OpenMP. The key contributions of this paper are as follows. First, we give parallelization of an emerging application using OpenMP. Second, we identify some performance penalties and give effective recipes. Considering the 2,200 nodes dataset, the optimizations enhance the parallel speedup up to $88 \%(2.94 / 1.56$ - 1), together with a $2 \mathrm{X}(14.6 / 7)$ sequential performance improvement. Third, we analyze impact patterns of different optimizations on sequential performance and parallel speedup.

The results from this paper emphasize the importance of a memory-friendly program to increase the parallel efficiency of a shared-memory multi-processor, especially the importance of control the random data layout caused by the dynamic memory allocation. At the same time, the efficiency of memory usage of STL vectors on SMP needs also be improved.

\section{References}

[1] Intel vtune performance analyzer. Intel Corporation. http://developer.intel.com/software/products/vtune/ index.htm.

[2] Pnl: Probabilistic network library. Intel Corporation. http://sourceforge.net/projects/openpnl/.

[3] Standard template library programmer's guide. SGI Corporation. http://www.sgi.com/tech/stl/.

[4] OpenMP $C$ and $C++$ Application Program Interface, 2.0 Edition. The OpenMP Architecture Review Board, Mar. 2002.

[5] D. Bacon, S. Graham, and O. Sharp. Compiler transformations for high performance computing. ACM Computing Surveys, 26(4):345-420, Dec. 1994.

[6] E. Berger, K. McKinley, R. Blumofe, and P. Wilson. Hoard: A scalable memory allocator for multithreaded applications, 2000. http://www.cs.umass.edu/ emery/hoard/.

[7] S. Borkar, P. Dubey, and et al. Platform 2015: Intel processor and platform evolution for the next decade, 2005. ftp://download.intel.com/technology/computing/ archinnov/platform2015/download/Platform_2015.pdf.

[8] D. Heckerman. A tutorial on learning with bayesian networks, 1996. ftp://ftp.research.microsoft.com/pub/tr/tr-95-06.pdf.

[9] L. Liu, W. Hu, C. Lai, H. Jiang, W. Chen, W. Zheng, and Y. Zhang. Parallel module network learning on distributed memory multiprocessors. In 2005 International Conference on Parallel Processing Workshops (ICPPW'05), pages 129-134, 2005.

[10] K. Murphy. A brief introduction to graphical models and bayesian networks, 1998. http://www.cs.ubc.ca/ ${ }^{\sim}$ murphyk/Bayes/bayes.html.

[11] E. Segal, D. Pe'er, A. Regev, D. Koller, and N. Friedman. Learning module networks. The Journal of Machine Learning Research, 6:557-588, Sept. 2005.

[12] X. Xu, L. Wang, and D. Ding. Learning module networks from genome-wide location and expression data. FEBS Lett., 578(3):297-304, Dec. 2004. 